\title{
Will long term oncologic follow-up make the case for robotic assisted radical cystectomy?
}

\author{
Michael D. Gross, Bashir Al Hussein Al Awamlh, Jim C. Hu \\ Department of Urology, New York Presbyterian Hospital, Weill Cornell Medicine, New York, NY, USA \\ Correspondence to: Jim C. Hu, MD, MPH. Department of Urology, New York Presbyterian Hospital, Weill Cornell Medicine, 525 East 68th Street, \\ Starr 946, New York, NY 10065, USA. Email: Jch9011@med.cornell.edu. \\ Provenance: This is an invited Editorial commissioned by Section Editor Xiao Li (Department of Urology, Jiangsu Cancer Hospital \& Jiangsu \\ Institute of Cancer Research \& Nanjing Medical University Affiliated Cancer Hospital, Nanjing, China). \\ Comment on: Bochner BH, Dalbagni G, Marzouk KH, et al. Randomized Trial Comparing Open Radical Cystectomy and Robot-assisted \\ Laparoscopic Radical Cystectomy: Oncologic Outcomes. Eur Urol 2018;74:465-71.
}

Submitted Oct 20, 2018. Accepted for publication Nov 09, 2018.

doi: $10.21037 /$ tau.2018.11.01

View this article at: http://dx.doi.org/10.21037/tau.2018.11.01

The use of robot-assisted radical cystectomy (RARC) has increased steadily over the last 15 years (1). The impetus for adopting minimally invasive surgery is based on decreased perioperative morbidity and length of stay compared to the gold standard, open radical cystectomy (ORC). However, the most important aspect in cancer surgery adoption is oncological outcome and, until recently, there has been a paucity of prospective randomized controlled trails (RCTs) comparing RARC and ORC. Following their initial publication that pertained to perioperative outcomes (2), Bochner et al. report the oncological outcomes of a singlecenter, prospective RCT which included 118 patients with median follow-up of 5 years (3). In this study, the authors did not find a significant difference in recurrence-free, cancerspecific and overall survival between RARC and ORC.

Patients were randomized with allocation concealment to either RARC or ORC with pelvic lymphadenectomy and open/extracorporeal urinary diversion between 2010 and 2013. Baseline characteristics, including age and use of neoadjuvant chemotherapy were similar in both groups. Patients with cT4 disease were excluded from the analysis, however the pathologic staging included those with pT4 disease $(6.9 \%$ in ORC and $8.3 \%$ in RARC) and was distributed equally.

Bochner et al. reported similar oncological outcomes between both groups of patients; there were no significant differences in disease-specific or recurrence-free survival ( $\mathrm{P}=0.4$ for both), as well as all-cause mortality $(\mathrm{P}=0.8)$. In their analysis, disease recurrence was subdivided into local, abdominal, or distal. When comparing patterns of recurrence in that distribution there were no significant differences between open and robotic cases. However, when pelvic and abdominal recurrences were grouped as "locoregional" recurrences, the ORC group had a significantly lower rate of recurrence in this distribution (sHR: 0.34; 95\% CI: 0.12-0.93; P=0.035). Conversely, while not statistically significant, the ORC group had a higher rate of distant metastases (sHR: 2.21; 95\% CI: 0.96-5.12; $\mathrm{P}=0.064)$. It is important to keep in mind that the trial was designed and powered to assess complication rates between RARC and ORC, therefore the oncological outcomes are secondary and should be interpreted with caution.

Previous studies have often suggested that 2 years is sufficient to detect $>80 \%$ of recurrences (4). This study distinguishes itself based on the relatively long-term followup (median 4.9 years) as it includes recurrence data from patients past the 2-year follow-up mark. Oftentimes, patients with advanced disease preferentially undergo ORC due to the complexity of the procedure. The study of interest maintained a balance in disease stage between both groups, however, the entire cohort is skewed towards lower stage disease. This distribution does not reflect the national or their own institutional trends as noted by the authors themselves, which further limits what can be deduced about recurrence and survival in those with advanced disease opting to undergo RARC.

These results are comparable to other important retrospective and prospective studies; including a study 
that included 2,187 patients from 17 countries by the International Robotic Cystectomy Consortium (IRCC) (5) and the Randomized Open versus Robotic Cystectomy (RAZOR) phase 3, non-inferiority trial that analyzed 302 patients from 15 academic centers across the U.S (6). The IRCC found recurrence free, cancer-specific, and overall survival to be $67 \%, 75 \%$, and $50 \%$, respectively with a median follow-up of 5.6 years. At the 2-year endpoint of the RAZOR trial, progression-free survival in the RARC group compared with ORC $\left(72.3 \%\right.$ vs. $71.6 \%$; $\left.\mathrm{P}_{\text {noninferiority }}=0.001\right)$ was also similar. In line with the aforementioned studies, the study by Bochner et al. reports a risk of recurrence of $36 \%$ and $41 \%$ at 5 years for RARC and ORC, respectively (difference: $-5.2 \%$; 95\% CI: $-25 \%$ to $14 \%$ ).

Positive surgical margin (PSM) rate for RARC relative to ORC was $6 \%$ vs. $5 \%(\mathrm{P}=0.59)$ in the RAZOR trial compared to $3.6 \%$ vs. $4.8 \%$ in the Bochner et al. study. Additionally, the IRCC reported PSM in $8 \%$ of their population. Given that PSM may correlate with higher stage disease, the higher rate observed in the IRCC and RAZOR data may be reflective of the larger percentage of pT4 disease in that cohort ( $9 \%$ in IRCC and $8-11 \%$ in RAZOR). In addition, the heterogeneity of PSM rates in RARC may indeed be attributable to the learning curve and the added difficulty of lack of tactile feedback during RARC (7).

In contrast to the Bochner et al. study, the RAZOR trial found no significant difference in local or distal recurrence between RARC and ORC ( $4 \%$ vs. $3 \% ; \mathrm{P}=0.54$, and $22 \%$ vs. $23 \%$, respectively). The definition of local recurrence in the RAZOR trial included recurrence in the cystectomy bed, pelvic lymphadenectomy template or abdominal wall/port site, whereas bowel recurrence and peritoneal carcinomatosis were considered distal recurrences. In the RAZOR trial, there were no significant differences found in rates of peritoneal carcinomatosis (two in RARC, one in ORC) and abdominal wall recurrence (one case in the ORC group and none in the RARC). Interestingly, Bochner et al. note an uncommon pattern of recurrence only in the RARC group with five cases involving the abdominal wall, excluding port sites, and three with rectosigmoid invasion. This rate is higher than previously published RARC $(8,9)$ and ORC series (10). The cause of uncommon recurrence patterns has been proposed to be attributable to pneumoperitoneum, tumor spillage, and inadequate resection (11) or may simply be explained by tumor stage (12). Albeit the difference in methodology and reporting of recurrence patterns, the incidence of atypical recurrences is low, and in each of the aforementioned studies remains comparable to the comparative ORC group.

While this recent analysis serves as a valuable contribution to the cache of randomized trials comparing RARC and ORC, it has several limitations; the longterm oncologic outcome data is underpowered to detect differences in overall recurrence trends and does not represent advanced stage cystectomy. Additionally, both this and the RAZOR trial perform exclusively extracorporeal urinary diversion, despite the growing interest in intracorporeal diversion in most high-volume centers (13). Furthermore, while the prescription of chemotherapy was similar between both groups, it was not standard and its use may obviate differences in post-surgical risk of recurrence.

The question that robotic approach might provide better oncological outcomes still remains under investigation. However, certain perioperative aspects have been reproduced in the literature, such as its positive impact on length of stay and post-operative blood loss. Of note, the adoption of enhanced recovery after surgery protocols may further narrow the margin between both techniques. Bochner et al. have made a substantial contribution in providing long term, randomized prospective follow up data to show no difference in RARC vs. ORC oncologic outcomes; such studies are crucial in advancing our understanding on the safety of RARC in order to provide the best patient care.

\section{Acknowledgements}

This article was supported by The Frederick J. and Theresa Dow Wallace Fund of the New York Community Trust.

\section{Footnote}

Conflicts of Interest: The authors have no conflicts of interest to declare.

\section{References}

1. Hu JC, Chughtai B, O'Malley P, et al. Perioperative Outcomes, Health Care Costs, and Survival After Robotic-assisted Versus Open Radical Cystectomy: A National Comparative Effectiveness Study. Eur Urol 2016;70:195-202.

2. Bochner BH, Dalbagni G, Sjoberg DD, et al. Comparing Open Radical Cystectomy and Robot-assisted Laparoscopic Radical Cystectomy: A Randomized Clinical Trial. Eur Urol 2015;67:1042-50. 
3. Bochner BH, Dalbagni G, Marzouk KH, et al.

Randomized Trial Comparing Open Radical Cystectomy and Robot-assisted Laparoscopic Radical Cystectomy: Oncologic Outcomes. Eur Urol 2018;74:465-71.

4. Tyritzis SI, Hosseini A, Collins J, et al. Oncologic, functional, and complications outcomes of robot-assisted radical cystectomy with totally intracorporeal neobladder diversion. Eur Urol 2013;64:734-41.

5. Raza SJ, Wilson T, Peabody JO, et al. Long-term oncologic outcomes following robot-assisted radical cystectomy: results from the International Robotic Cystectomy Consortium. Eur Urol 2015;68:721-8.

6. Parekh DJ, Reis IM, Castle EP, et al. Robot-assisted radical cystectomy versus open radical cystectomy in patients with bladder cancer (RAZOR): an open-label, randomised, phase 3, non-inferiority trial. Lancet 2018;391:2525-36.

7. Snow-Lisy DC, Campbell SC, Gill IS, et al. Robotic and laparoscopic radical cystectomy for bladder cancer: longterm oncologic outcomes. Eur Urol 2014;65:193-200.

8. Collins JW, Hosseini A, Adding C, et al. Early Recurrence Patterns Following Totally Intracorporeal Robot-assisted Radical Cystectomy: Results from the EAU Robotic

Cite this article as: Gross $\mathrm{MD}, \mathrm{Al}$ Hussein $\mathrm{Al}$ Awamlh $\mathrm{B}$, $\mathrm{Hu}$ JC. Will long term oncologic follow-up make the case for robotic assisted radical cystectomy? Transl Androl Urol 2018;7(Suppl 6):S696-S698. doi: 10.21037/tau.2018.11.01
Urology Section (ERUS) Scientific Working Group. Eur Urol 2017;71:723-6.

9. Hussein AA, Saar M, May PR, et al. Early Oncologic Failure after Robot-Assisted Radical Cystectomy: Results from the International Robotic Cystectomy Consortium. J Urol 2017;197:1427-36.

10. Kim B, Choi HJ, Kim MH, et al. Recurrence patterns of bladder transitional cell carcinoma after radical cystectomy. Acta Radiol 2012;53:943-9.

11. Nguyen DP, Al Hussein Al Awamlh B, Wu X, et al. Recurrence patterns after open and robot-assisted radical cystectomy for bladder cancer. Eur Urol 2015;68:399-405.

12. Niegisch G, Nini A, Michalski R, et al. Comparison of 2-Year Oncological Outcome and Early Recurrence Patterns in Patients with Urothelial Bladder Carcinoma Treated with Open or Robot-Assisted Radical Cystectomy with an Extracorporeal Urinary Diversion. Urol Int 2018;101:224-31.

13. Hussein AA, Saar M, May PR, et al. Early Oncologic Failure after Robot-Assisted Radical Cystectomy: Results from the International Robotic Cystectomy Consortium. J Urol 2017;197:1427-36. 